\title{
Production of the hidden charm state $X(3872)$ and of $\psi(2 S)$ with the ATLAS detector
}

\author{
James Broughton, on behalf of the ATLAS Collaboration \\ University of Birmingham (GB) \\ E-mail: j.broughton@cern.ch
}

Differential cross sections are presented for the prompt and non-prompt production of the hiddencharm state $X(3872)$ and $\psi(2 S)$, in the decay mode $J / \psi \pi^{+} \pi^{-}$, measured at $\sqrt{s}=8 \mathrm{TeV}$ by the ATLAS detector at LHC. In the non-prompt production, the data suggest the presence of a shortlived component, which may be due to $B_{c}$ decays. The dipion invariant masses in both $X(3872)$ and $\psi(2 S)$ are measured and compared to theoretical predictions.

25th International Workshop on Deep Inelastic Scattering and Related Topics

3-7 April 2017

University of Birmingham, Birmingham, UK 


\section{Introduction}

The hidden-charm state $X(3872)$ was discovered by the Belle Collaboration in 2003 through its decay to $J / \psi \pi^{+} \pi^{-}$[1]. Its existence was confirmed soon after by other experiments, including $\mathrm{CDF}$ who constrained the possible quantum numbers $J^{P C}$ to be either $J^{P C}=1^{++}$or $2^{-+}[2,3]$. LHCb was the first experiment at the LHC to observe the $X(3872$ ) [4], which confirmed its quantum numbers to be $1^{++}$[5]. The $X(3872)$ has a mass close to the $D^{0} \bar{D}^{* 0}$ threshold and, as a result, the state was initially hypothesised to be a $D^{0} \bar{D}^{* 0}$ molecule with a very small binding energy [6]. CMS performed a cross-section measurement of promptly produced $X(3872)$ [7] as a function of $p_{T}$, which showed the non-relativistic QCD (NRQCD) prediction [8] for prompt $X(3872)$ production, assuming a $D^{0} \bar{D}^{* 0}$ molecule, was too high. The current interpretation of the state is a mixed $\chi_{c 1}(2 P)-D^{0} \bar{D}^{* 0}$ state, where the $X(3872)$ is produced predominantly through its $\chi_{c 1}(2 P)$ component. CMS data show a good agreement with this new model [9].

The ATLAS collaboration [10] previously reported evidence for the $X(3872)$ state while measuring the cross section of prompt and non-prompt $\psi(2 S)$ meson production in the $J / \psi \pi^{+} \pi^{-}$decay channel with 2011 data at $\sqrt{s}=7 \mathrm{TeV}$ [11]. ATLAS has performed an extension to this analysis using $11.4 \mathrm{fb}^{-1}$ of $\sqrt{s}=8 \mathrm{TeV}$ proton-proton collision data [12].

\section{Event Selection}

This analysis searches for $X(3872)$ and $\psi(2 S)$ decaying to $J / \psi \pi^{+} \pi^{-}$, where the $J / \psi$ meson decays into a muon pair. Events used in this analysis are triggered by a pair of muons successfully fitted to a common vertex. Each muon candidate reconstructed offline must have good spatial trigger-object matching that satisfies $\Delta R=\sqrt{(\Delta \phi)^{2}+(\Delta \eta)^{2}}<0.01$, where $\Delta R$ is the angular distance between the momenta of the muon candidate and the trigger object, $\phi$ is the azimuthal angle around the $z$-axis and $\eta$ is the pseudorapidity defined in terms of the polar angle $\theta$ as $\eta=-\ln \tan (\theta / 2)$. Events where two oppositely-charged muon candidates are reconstructed, with pseudorapidity $\left|\eta^{\mu}\right|<2.3$ and transverse momenta $p_{T}^{\mu}>4 \mathrm{GeV}$, are kept only if the invariant mass of the dimuon system falls within $\pm 120 \mathrm{MeV}$ of the mass of the $J / \psi$ meson, $m(J / \psi)=3096.9$ $\mathrm{MeV}$ [13]. The dimuon invariant mass is shown in Figure 1(a).

The two muon tracks are fitted to a common vertex with a loose cut on fit quality, $\chi^{2}<200$. The dimuon invariant mass is then constrained to the $J / \psi$ mass, and the four-track vertex fit of the two muon tracks and pairs of opposite-charged non-muon tracks is performed to find the $J / \psi \pi^{+} \pi^{-}$ candidates. The two non-muon tracks are assigned pion masses, and are required to satisfy $p_{T}^{\pi}>$ $0.6 \mathrm{GeV}$ and $\left|\eta^{\pi}\right|<2.4$. To further suppress the background, the four-track candidates with $\chi^{2}$ probability $P\left(\chi^{2}\right)<4 \%$ are discarded. The opening angle $\Delta R\left(J / \psi, \pi^{+}\right)$must be less than 0.5 and the mass difference between the four track candidate and the combination of $m(J / \psi)$ plus $m\left(\pi^{+} \pi^{-}\right)$must be less than $300 \mathrm{MeV}$.

Four-track candidates passing the above selection and lying within the rapidity region $|y|<$ 0.75 are used in the analysis. The four-track invariant mass distribution is shown in Figure 1(b). These selection requirements are found to be $>90 \%$ efficient for the $\psi(2 S)$ and $X(3872)$ decays in signal Monte-Carlo, while significantly suppressing the combinatorial background. 


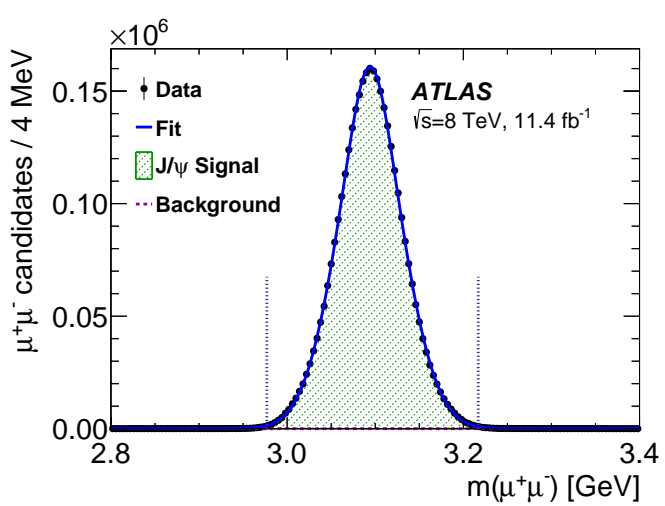

(a)

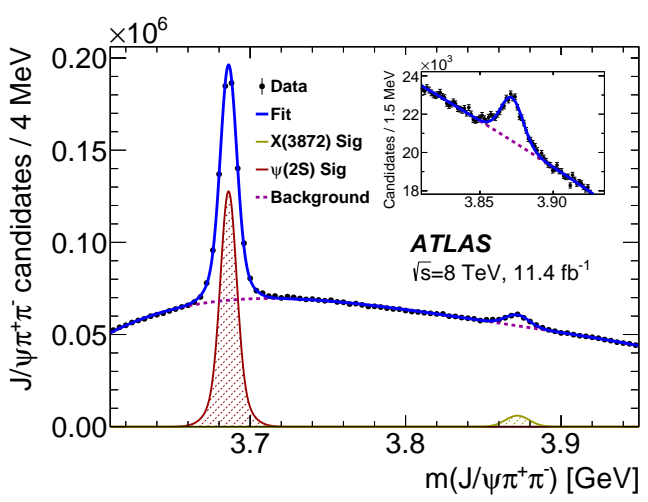

(b)

Figure 1: (a) Invariant mass distribution of the $J / \psi$ candidates satisfying all selection criteria except the $J / \psi$ mass window requirement [12].(b) Invariant mass of the selected $J / \psi \pi^{+} \pi^{-}$candidates after selection requirements [12].

\section{Analysis Method}

Candidates that pass the selection criteria are weighted based on acceptance, reconstruction and trigger efficiency. Events are divided into five $p_{T}$ bins and four effective pseudo-proper lifetime $\tau$ bins, where $\tau=L_{x y} m / p_{T}$ with $L_{x y}=\vec{L} \cdot \vec{p}_{T} / p_{T}$ and $\vec{L}$ is the vector pointing from the primary $p p$ collision vertex to the $J / \psi \pi^{+} \pi^{-}$vertex. Separation based on the pseudo-proper lifetime distinguishes the prompt production of the $\psi(2 S)$ and $X(3872)$ states from the non-prompt production occurring via the decays of long-lived particles such as $b$-hadrons.

For each $p_{T}$ and lifetime bin, a minimum $\chi^{2}$ fit in the $J / \psi \pi^{+} \pi^{-}$invariant mass is performed to determine the signal yields of the $\psi(2 S)$ and $X(3872)$ states. For each $p_{T}$ bin, the yields in individual lifetime windows are fitted separately for $\psi(2 S)$ and $X(3872)$.

\section{Lifetime Fits}

The probability density function (PDF) describing the dependence of $\psi(2 S)$ and $X(3872)$ signal yields on the pseudo-proper lifetime $\tau$ is a superposition of prompt (P) and non-prompt (NP) components:

$$
F^{i}(\tau)=\left(1-f_{N P}^{i}\right) F_{P}^{i}(\tau)+f_{N P}^{i} F_{N P}^{i}(\tau),
$$

where $f_{N P}$ is the non-prompt fraction, while $i$ stands for either $\psi(2 S)$ or $X(3872)$ signals. The term $f_{N P}$ is measured in each $p_{T}$ bin, separately for each $i$. The prompt signal $F_{P}^{i}$ is described by a lifetime resolution function determined from the data. For the non-prompt signal $F_{N P}^{i}$, a single onesided exponential is convolved with the resolution function, with a single "effective pseudo-proper lifetime" fitted to the data. This approach is known as the single-lifetime fit.

Figure 2(a) shows the measured effective pseudo-proper lifetimes $\tau_{\text {eff }}$ for non-prompt $X$ (3872) and $\psi(2 S)$ decays in bins of $p_{T}$. While for $\psi(2 S)$ the fitted values of $\tau_{\text {eff }}$ are measured to be around $1.45 \mathrm{ps}$ in all $p_{T}$ bins, the signal from $X(3872)$ at low $p_{T}$ tends to have shorter lifetimes, suggesting there could be a different production mechanism contributing at low $p_{T}$.

In Figure 2(b) the measured ratio of non-prompt production cross sections of $X(3872)$ and $\psi(2 S)$ is plotted as a function of $p_{T}$ with a kinematic template fitted. The template assumes that 


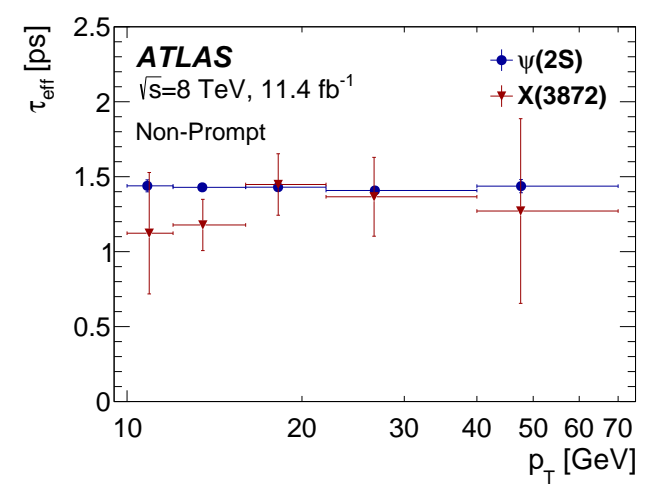

(a)

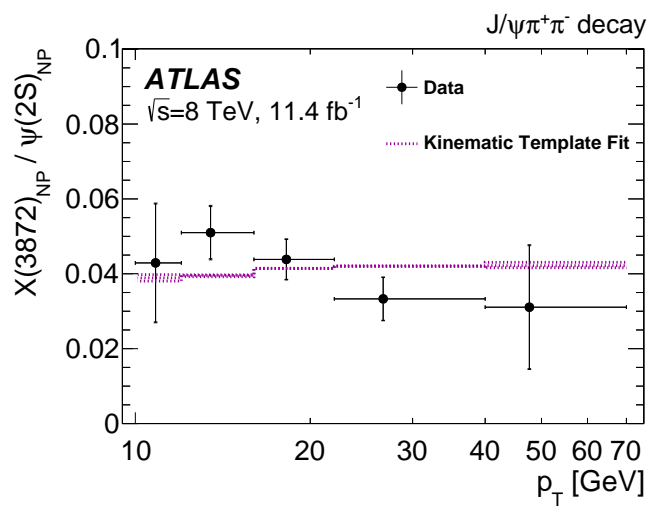

(b)

Figure 2: (a) Measured effective pseudo-proper lifetimes for non-prompt $X(3872)$ and $\psi(2 S)$ [12]. (b) Ratio of non-prompt production cross sections, $X(3872) / \psi(2 S)$ in the single lifetime fit model [12].

non-prompt $\psi(2 S)$ and $X(3872)$ are produced from the same admixture of parent $b$-hadrons and, therefore, implies the same lifetimes for $\psi(2 S)$ and $X(3872)$ in each $p_{T}$ bin. This allows determination of the ratio of the average branching fractions to be:

$$
R_{B}^{1 L}=\frac{\operatorname{Br}(B \rightarrow X(3872)+\text { any }) \operatorname{Br}\left(X(3872) \rightarrow J / \psi \pi^{+} \pi^{-}\right)}{B r(B \rightarrow \psi(2 S)+\text { any }) \operatorname{Br}\left(\psi(2 S) \rightarrow J / \psi \pi^{+} \pi^{-}\right)}=(3.95 \pm 0.32(\text { stat }) \pm 0.08(\text { sys })) \% .
$$

An alternative lifetime model, also implemented in this analysis, allows for two non-prompt contributions with distinctly different effective lifetimes (the "two-lifetime fit"). The non-prompt component is represented as a sum of short-lived (SL) and long-lived (LL) components:

$$
F_{N P}^{i}(\tau)=\left(1-f_{S L}^{i}\right) F_{L L}(\tau)+f_{S L}^{i} F_{S L}(\tau),
$$

where $f_{S L}^{i}$ is the fraction of SL within non-prompt. The statistical power of the data does not allow determination of two free lifetimes and so these are fixed, with $f_{S L}^{i}$ left free in the fit. The LL component is assumed to originate from the usual admixture of $B^{ \pm}, B^{0}, B_{s}$ mesons and $b$-baryons, while any SL part would be due to the contribution of $B_{c}^{ \pm}$mesons. The lifetimes depend on the parent's lifetime and decay kinematics. The term $\tau_{L L}$ is determined from fits to $\psi(2 S)$ and allows for some SL contribution, $\tau_{L L}=1.45 \pm 0.05 \mathrm{ps}$. The $B_{c}$ decay kinematics are varied in the simulation to alter the expected SL lifetime; the mean of the variation is taken to obtain $\tau_{S L}=0.40 \pm 0.05 \mathrm{ps}$. The measured ratio of long-lived $X(3872)$ to long-lived $\psi(2 S)$ is fitted with the kinematic template as described above, to obtain

$$
R_{B}^{2 L}=\frac{B r(B \rightarrow X(3872)+\text { any }) \operatorname{Br}\left(X(3872) \rightarrow J / \psi \pi^{+} \pi^{-}\right)}{B r(B \rightarrow \psi(2 S)+\text { any }) \operatorname{Br}\left(\psi(2 S) \rightarrow J / \psi \pi^{+} \pi^{-}\right)}=(3.57 \pm 0.33(\text { stat }) \pm 0.11(\text { sys })) \% .
$$

This value of $R_{B}$ is lower than the corresponding result from the single lifetime model, but both values are significantly smaller than the $18 \%$ expected from an estimate using Tevatron data [8] and the world average values for the branching fractions: $\operatorname{Br}(B \rightarrow \psi(2 S))=(3.07 \pm 0.21) \times 10^{-3}$, $\operatorname{Br}\left(\psi(2 S) \rightarrow J / \psi \pi^{+} \pi^{-}\right)=(34.46 \pm 0.30) \%$ [14].

The fraction of non-prompt $X(3872)$ from short-lived sources is found to be: 


$$
\frac{\sigma\left(p p \rightarrow B_{c}\right) \operatorname{Br}\left(B_{c} \rightarrow X(3872)\right)}{\sigma(p p \rightarrow \text { non-prompt } X(3872))}=(25 \pm 13(\text { stat }) \pm 2(\text { sys }) \pm 5(\text { spin })) \% .
$$

The measured differential cross section (times the relevant branching fractions) for prompt production of $\psi(2 S)$ is modelled fairly well by the NLO NRQCD model [15] with long-distance matrix elements (LDMEs) determined from the Tevatron data. Similarly, the differential cross section for prompt production of $X(3872)$ is modelled well by the NLO NRQCD model when assuming the $X(3872)$ state is a mixture of $\chi_{c 1}(2 P)-D^{0} \bar{D}^{* 0}$ with the $\chi_{c 1}(2 P)$ coupling responsible for production. In the non-prompt case, the $\psi(2 S)$ differential cross section is described well by the FONLL model [16], whereas this model overshoots the data in the $X(3872)$ case and increases with $p_{T}$.

The non-prompt fractions for $\psi(2 S)$ and $X(3872)$ are shown in Figure 3. In the case of $\psi(2 S)$, $f_{N P}$ increases with $p_{T}$ with good agreement between ATLAS and CMS. $X(3872)$ dimuon decays show no sizeable dependence on $p_{T}$, agreeing with the CMS result obtained at $\sqrt{s}=7 \mathrm{TeV}$ [17].

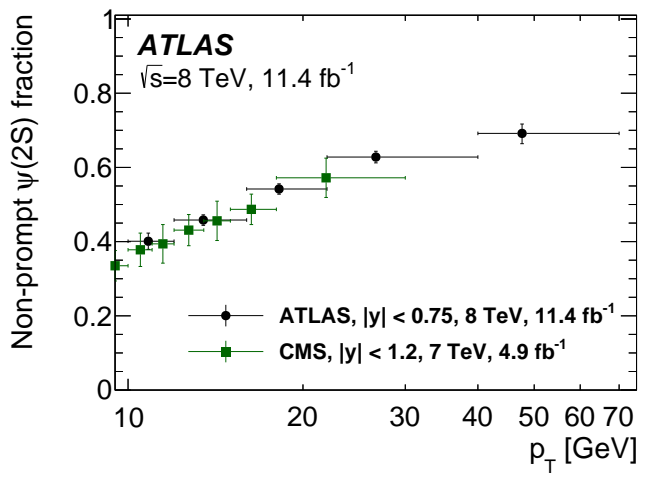

(a)



(b)

Figure 3: Measured non-prompt fractions for (a) $\psi(2 S)$ and (b) $X(3872)$ production compared to CMS results at $\sqrt{s}=7 \mathrm{TeV}[12]$.

\section{Dipion invariant mass spectra}

The invariant mass distributions of the dipion system in the decays of $\psi(2 S)$ and $X(3872)$ into $J / \psi \pi^{+} \pi^{-}$have been measured. For $\psi(2 S)$, shown in Figure 4(a) this peaks at high masses and is fitted with a Voloshin-Zakharov function:

$$
\frac{1}{\Gamma} \frac{d \Gamma}{d m_{\pi \pi}} \propto\left(m_{\pi \pi}^{2}-\lambda m_{\pi}^{2}\right)^{2} \times \mathrm{PS}
$$

where PS stands for the dipion phase-space. The fitted value is $\lambda=4.16 \pm 0.06$ (stat) \pm 0.03 (syst), in agreement with $\lambda=4.35 \pm 0.18$ measured by BES [18], and $\lambda=4.46 \pm 0.25$ measured by LHCb [19]. In Figure 4(b), the normalised differential decay width of the $X(3872)$ state is shown. The shaded blue histogram is obtained from simulations, assuming the dipion system in the $X(3872) \rightarrow J / \psi \pi^{+} \pi^{-}$is produced purely via the $\rho^{0}$ meson, and is in good agreement with the data. In both decays the $m_{\pi \pi}$ spectrum strongly disfavours the dipion phase-space distribution, shown by the red shaded area. 




(a)

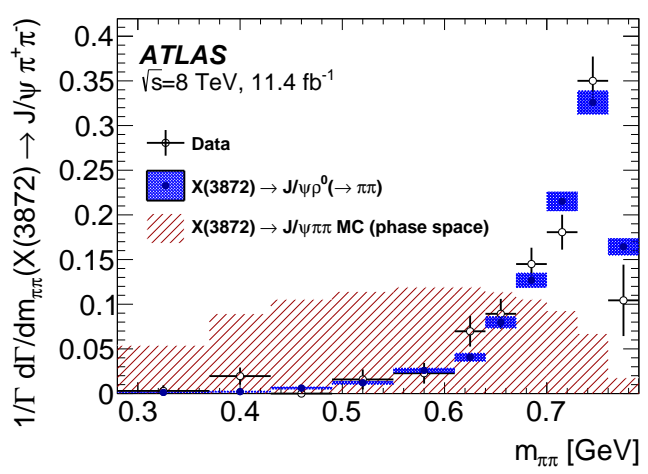

(b)

Figure 4: Normalised differential decay width as a function of invariant mass of (a) $\psi(2 S)$ and (b) $X(3872)$ decays [12].

\section{Summary}

ATLAS has measured the hidden-charm state $X(3872)$ and $\psi(2 S)$ using $11.4 \mathrm{fb}^{-1}$ of $\sqrt{s}=8$ $\mathrm{TeV}$ proton-proton collision data showing good agreement with the interpretation of the $X(3872)$ as a mixture of $\chi_{c 1}(2 P)-D^{0} \bar{D}^{* 0}$. The measurements are consistent with similar results published by the CMS collaboration.

\section{References}

[1] Belle Collaboration, Phys. Rev. Lett. 91 (2003) 262001, [hep-ex/ 0309032 ].

[2] CDF Collaboration, Phys. Rev. Lett. 93 (2004) 072001, [hep-ex/ 0312021$].$

[3] CDF Collaboration, Phys. Rev. Lett. 98 (2007) 132002, [hep-ex/0612053].

[4] LHCb Collaboration, Eur. Phys. J. C 72 (2015) 1972, [1112.5310 [hep-ex] ].

[5] LHCb Collaboration, Phys. Rev. D 92 (2015) 011102, [1504.06339 [hep-ex]].

[6] Tomaradze, A. et al. Phys. Rev. Lett. 91 (2015) 011102, [1501.01658 [hep-ex] ].

[7] CMS Collaboration, JHEP 04 (2013) 154, [1302.3968 [hep-ex] ].

[8] Artoisenet, P. and Braaten, E., Phys. Rev. D 81 (2010) 114018, [0911.2016 [hep-ph] ].

[9] Meng, C., Han, H. and Chao, K., X(3872) and its production at hadron colliders, (2013), [1304.6710 [hep-ph]].

[10] ATLAS Collaboration, JINST 3 (2008) S08003

[11] ATLAS Collaboration, JHEP 09 (2014) 079, [1407.5532 [hep-ex] ].

[12] ATLAS Collaboration, JHEP 01 (2017) 117, [1610.09303 [hep-ex] ].

[13] Particle Data Group, Chinese Physics C 38 (2014) 090001.

[14] Particle Data Group, Chinese Physics C 40 (2016) 100001.

[15] ATLAS Collaboration, Phys. Rev. D 90 (2014) 052007, [1407.1796 [hep-ex]].

[16] Cacciari, M. et al., JHEP 10 (2012) 137, [1205.6344 [hep-ph] ].

[17] CMS Collaboration, JHEP 02 (2012) 011, [1111.1557 [hep-ex] ].

[18] BES Collaboration, Phys. Rev. D 62 (2000) 032002, [hep-ex/ 9909038 ].

[19] LHCb Collaboration, Eur. Phys. J. C 72 (2012) 2100, [1204 .1258 [hep-ex] ]. 